DOI:10.2478/rrlm-2019-0026

\title{
Magnesium isoglycyrrhizinate protects against concanavalin A-induced immunological liver injury in a mouse model
}

\author{
Zhengyan Jiang ${ }^{1}$, Liang Zheng ${ }^{1 *}$ \\ 1. Department of Spleen and Stomach Diseases, The Second Affiliated Hospital of Nanjing University \\ of Chinese Medicine, Jiangsu Provincial Second Hospital of Traditional Chinese Medicine, Nanjing, \\ Jiangsu Province, China
}

\begin{abstract}
Background: To evaluate the protective effects of magnesium isoglycyrrhizinate on a mouse model of concanavalin A (ConA)-induced immunological liver injury. Materials and Methods: Forty-eight mice were randomly divided into a normal control group, a model group, three dose groups of magnesium isoglycyrrhizinate (12.5, 25, $50 \mathrm{mg}$ ) $\mathrm{kg}$ ) and a dexamethasone group (2.5 $\mathrm{mg} / \mathrm{kg})$. Magnesium isoglycyrrhizinate was intraperitoneally injected for 5 consecutive days, and the model of immunological liver injury was established on the fifth day after caudal vein injection of ConA (20 mg/kg). Blood was collected to detect the activities of alanine transaminase (ALT) and aspartate transaminase (AST) as well as the levels of tumor necrosis factor- $\alpha(T N F-\alpha)$ and interferon- $\gamma(I F N-\gamma)$. The levels of neopterin (NP) and malondialdehyde (MDA) and the activities of myeloperoxidase (MPO) and superoxide dismutase (SOD) in liver tissues were measured, and histopathological changes were observed. Results: The serum levels of ALT and AST in the model group increased. Hepatic lobules had necrotic foci and inflammatory cell infiltration. The plasma levels of TNF- $\alpha$ and IFN- $\gamma$ increased. In liver tissues, the levels of NP, MDA and MPO rose, but that of SOD decreased. Magnesium isoglycyrrhizinate significantly attenuated the activities of ALT and AST $(P<0.05)$. Histopathological staining showed that inflammation of the liver was relieved significantly. Magnesium isoglycyrrhizinate also decreased the levels of NP, MDA and MPO in liver tissues $(P<0.05)$, raised that of $S O D$ and reduced the plasma levels of TNF- $\alpha$ and IFN- $\gamma(P<0.05)$. Conclusion: Magnesium isoglycyrrhizinate protected against ConA-induced immunological liver injury in mice, probably through immune regulation and antioxidation.
\end{abstract}

Keywords: immunological liver injury, magnesium isoglycyrrhizinate, immune regulation, antioxidation Received: 15 th October 2018; Accepted: 11 th April 2019; Published: $18^{\text {th }}$ June 2019

\footnotetext{
*Corresponding author: Liang Zheng, Department of Spleen and Stomach Diseases, The Second Affiliated Hospital of Nanjing University of Chinese Medicine, Jiangsu Provincial Second Hospital of Traditional Chinese Medicine, Nanjing, Jiangsu Province, China. E-mail: zhengliangdssd@yahoo.com
} 


\section{Introduction}

Hepatitis refers to a group of diseases caused by a variety of pathogenic factors which damage the liver and induce inflammation [1]. As a result, hepatocytes are destroyed, and the liver function is impaired, further leading to symptoms. Common pathogenic factors include viruses, bacteria, chemical toxicants, drugs, alcohol, etc. [2]. Immunological mechanisms play important roles in liver injury, which can reduce the release of inflammatory cytokines, and inhibit the expressions of adhesion molecules and up-regulation of death receptors [3]. Until now, there are no effective drugs for treating viral hepatitis, so integrative therapy is usually recommended. For instance, antiviral, antifibrotic and immunomodulatory therapies have been used [4]. Interferon- $\gamma$ (IFN- $\gamma$ ) can prevent virus from replicating in host hepatocytes to exert immunomodulatory effects [5]. However, its indications are limited, with apparent side effects also. The therapeutic effects of nucleoside analogues are stable, but long-term use easily causes drug resistance [6]. Studying the pathogenesis of liver diseases and screening drugs in clinical practice both rely on the establishment of animal models with similar pathological processes of liver diseases to those of human. The mouse model of concanavalin A (ConA)-induced acute liver injury, which is established through $\mathrm{T}$ lymphocyte mediation, has liver specificity, without damaging the heart, lung, spleen or kidney [7]. This model is suitable for studying the mechanisms of human viral hepatitis and autoimmune liver disease as well as for screening agents for liver injury therapy. As a fourth-generation glycyrrhizin preparation, magnesium isoglycyrrhizinate is a magnesium salt of 18- $\alpha$ glycyrrhizic acid stereo-isomer, which exhibits obvious anti-inflammatory and antioxidative activities and stabilizes the cell membrane [8]. Up to now, the effects of magnesium isoglycyrrhizinate on immunological liver injury have never been assessed experimentally. Thereby motivated, we herein established a mouse model of ConA-induced immunological liver injury to evaluate its therapeutic effects and the underlying mechanism, aiming to provide a novel strategy for clinically treating this disease.

\section{Materials and Methods}

\section{Animals}

This study has been approved by the ethics committee of our hospital, and great efforts have been made to minimize animal suffering. A total of 144 ICR mice of either sex with the age of 4-6 weeks old and the weight of 20-25 g were provided by Shanghai SLAC Laboratory Animal Co., Ltd. (China). They were kept in the experimental animal center of our hospital. The temperature was set at $(20 \pm 2)^{\circ} \mathrm{C}$, the humidity was $50 \%$, and the light/dark cycle was $12 \mathrm{~h} / 12$ h. Standard mouse feed was given, and the mice had free access to distilled water. The experiment was started one week after adaptation to the environment.

\section{Materials}

Magnesium isoglycyrrhizinate was purchased from Jiangsu Chia Tai Tianqing Pharmaceutical Group Co., Ltd. (China). ConA (molecular weight: 102000) and neopterin (NP) were bought from Sigma (USA). Myeloperoxidase (MPO), malondialdehyde (MDA) and superoxide dismutase (SOD) detection kits were obtained from Nanjing Jiancheng Bioengineering Institute (China). Tumor necrosis factor (TNF)- $\alpha$ and IFN- $\gamma$ ELISA kits were provided by Shenzhen Jingmei Biotech Co., Ltd. (China).

\section{Establishment of immunological liver injury model}

The mouse model of ConA-induced acute liver injury was established according to the method of Tiegs et al. [9]. Briefly, the mice were inject- 
ed once with $20 \mathrm{mg} / \mathrm{kg}$ ConA that had been dissolved in $200 \mu \mathrm{l}$ of sterile phosphate buffered saline (PBS) through the caudal vein.

\section{Experimental grouping and drug administra- tion}

The mice were randomly divided into 6 groups $(n=24)$ : a normal control group, a model group, a low-dose magnesium isoglycyrrhizinate group, a middle-dose magnesium isoglycyrrhizinate group, a high-dose magnesium isoglycyrrhizinate group and a dexamethasone $(2.5 \mathrm{mg} / \mathrm{kg})$ group. The doses of magnesium isoglycyrrhizinate were set at $12.5 \mathrm{mg} / \mathrm{kg} / \mathrm{d}, 25 \mathrm{mg} / \mathrm{kg} / \mathrm{d}$ and $50 \mathrm{mg} / \mathrm{kg} / \mathrm{d}$ respectively according to those for humans, and this drug was administered through intraperitoneal injection. Different doses of magnesium isoglycyrrhizinate were given for five consecutive days before ConA injection and once more $1 \mathrm{~h}$ after injection. The dexamethasone group was administered with dexamethasone once before and $1 \mathrm{~h}$ after ConA injection, respectively. The normal control group and the model group were both intraperitoneally injected with equal volumes of PBS. After modeling, the mice were fasted but given free access to water for $16 \mathrm{~h}$ and weighed, from which the eyeballs were disconnected to collect blood samples. Then they were dissected, and the livers were taken and stored prior to use.

\section{Detection of serum alanine transaminase (ALT) and aspartate transaminase (AST) ac- tivities}

The mice were anesthetized though intraperitoneal injection of $10 \%$ chloral hydrate $(0.04 \mathrm{ml} / \mathrm{g})$ $8 \mathrm{~h}$ after ConA injection into the caudal vein. The collected blood samples were centrifuged at $3000 \mathrm{r} / \mathrm{min}$ for $15 \mathrm{~min}$, and the supernatant was stored at $4{ }^{\circ} \mathrm{C}$ for detection. Serum ALT and AST activities were measured according to kits' instructions.

\section{Detection of plasma TNF- $\alpha$ and IFN- $\gamma$ levels}

TNF- $\alpha$ and IFN- $\gamma$ levels were determined by double-antibody sandwich ELISA. Briefly, kits were equilibrated at room temperature, and then samples or standards at different concentrations (100 $\mu 1 /$ well) were added to corresponding wells respectively. Afterwards, the wells were incubated in a $37^{\circ} \mathrm{C}$ incubator for $90 \mathrm{~min}$. The plates were washed 4 times. Except for blank wells, all the others were added a working solution of biotinylated antibody $(100 \mu \mathrm{l} /$ well $)$. Then the wells were incubated at $37^{\circ} \mathrm{C}$ for $60 \mathrm{~min}$. Subsequently, the plates were washed 4 times. Except for blank wells, all the others were added a working solution of enzyme conjugate $(100 \mu \mathrm{l} /$ well). Then the wells were sealed and incubated at $37^{\circ} \mathrm{C}$ for $30 \mathrm{~min}$. The plates were thereafter washed 4 times. Then color development reagent (100 $\mu 1 /$ well) was added, and the plates were incubated at $37^{\circ} \mathrm{C}$ in dark for $10-15 \mathrm{~min}$. Finally, stopping buffer $(100 \mu \mathrm{l} /$ well $)$ was added, and the optical density (OD) at $450 \mathrm{~nm}$ was measured immediately after mixing. A standard curve was plotted by subtracting the OD values of each standard and sample from that of zeroing well. The levels of TNF- $\alpha$ and IFN- $\gamma$ in sample were calculated according to corresponding standard curves.

\section{Pathological examination of liver tissues}

The mice were anesthetized with $10 \%$ chloral hydrate by intraperitoneal injection $8 \mathrm{~h}$ after injection of ConA into the caudal vein. After blood was collected, the mice were fixed, and the abdominal wall was dissected along the midline. The abdominal cavity was exposed, and the liver was carefully separated. After washing with icecold normal saline, the liver morphology was observed with naked eyes. A part of the right liver lobe was cut off and fixed in $10 \%$ formaldehyde solution. The tissue was embedded in 
paraffin, sectioned into $4 \mu \mathrm{m}$-thick and stained with hematoxylin-eosin (HE). Pathohistological changes were observed by light microscopy.

\section{Detection of MDA, SOD and MPO levels in liver homogenate}

Mouse liver homogenate was prepared in icecold PBS by grinding the collected liver with a grinder. MDA, SOD and MPO levels in the liver homogenate were detected by the thiobarbituric acid method, the xanthine oxidase method and the tetramethyl benzidine method, respectively.

\section{Detection of NP level in liver homogenate}

NP level in liver homogenate was detected by high performance liquid chromatography. Liver homogenate $(0.45 \mathrm{ml})$ was mixed with $0.05 \mathrm{ml}$ of double-distilled water, mixed with $0.15 \mathrm{ml}$ of $300 \mathrm{~g} / \mathrm{L}$ trichloroacetic acid through vortexing for $30 \mathrm{~s}$, placed in the dark at room temperature for $10 \mathrm{~min}$, and centrifuged at $12,000 \mathrm{r} / \mathrm{min}$ at $4^{\circ} \mathrm{C}$ for $15 \mathrm{~min}$. Afterwards, $150 \mu \mathrm{l}$ of supernatant was collected, mixed with $100 \mu$ of alkali binding agent, and left still at room temperature for $5 \mathrm{~min}$ in the dark.

Chromatographic conditions: Waters 474 fluorescence detector; column: Kromasil-C8 (Dalian Elite Analytical Instruments Co., Ltd., China); mobile phase: methanol: $10 \mathrm{mmol} / \mathrm{L} \mathrm{KH}_{2} \mathrm{PO}_{4}=$ 10:90 (v/v); flow rate: $0.8 \mathrm{ml} / \mathrm{min}$; fluorescence detection wavelengths: $\mathrm{Ex}=360 \mathrm{~nm}, \mathrm{Em}=440$ $\mathrm{nm}$; column temperature: $20^{\circ} \mathrm{C}$; injection volume: $10 \mu$ l.

\section{Statistical analysis}

All data were analyzed by SPSS16.0 software and expressed as mean \pm standard deviation. Analysis of variance was performed, and the data with variance heterogeneity were subjected to the Dunnett's t test. $\mathrm{P}<0.05$ was considered statistically significant.

\section{Results}

\section{Effects of magnesium isoglycyrrhizinate on serum ALT and AST levels}

Serum ALT and AST levels are clinically sensitive indices reflecting the degrees of hepatocyte injury and necrosis. The serum ALT and AST levels of the model group were significantly higher than those of the normal control group $(\mathrm{P}<0.01)$. Prophylactic administration of magnesium isoglycyrrhizinate significantly reversed increase in the two levels $(\mathrm{P}<0.01 ; \mathrm{P}<0.05)$, so the liver cell injury and necrosis were milder than those of the model group. The effects of high-dose magnesium isoglycyrrhizinate were comparable to those of positive control drug dexamethasone (Table 1).

\section{Effects of magnesium isoglycyrrhizinate on liver pathology}

In the normal control group, hepatic lobules were intact and hepatocytes were arranged radially with the central vein as the center (Figure 1A). In the model group, most hepatocytes in liver lobules were swollen, with obvious punctate and necrotic foci. The necrotic foci were infiltrated

Table 1. Effects of magnesium isoglycyrrhizinate on serum ALT and AST levels

\begin{tabular}{lccc}
\hline Group & Dose (mg/kg) & ALT (U/L) & AST (U/L) \\
\hline Normal control & & $38.74 \pm 5.05^{* *}$ & $131.79 \pm 11.86^{* *}$ \\
\hline Model & & $341.19 \pm 10.70$ & $477.71 \pm 11.13$ \\
\hline Magnesium isoglycyrrhizinate & 50 & $126.73 \pm 13.52^{* *}$ & $227.50 \pm 11.65^{* *}$ \\
\hline & 25 & $156.78 \pm 11.63^{* *}$ & $279.39 \pm 11.24^{* *}$ \\
\hline Dexamethasone & 12.5 & $212.40 \pm 11.39^{*}$ & $336.0 \pm 12.51^{*}$ \\
\hline
\end{tabular}

Compared with model group, ${ }^{*} \mathrm{P}<0.05$, ${ }^{*} * \mathrm{P}<0.01$. 
with considerable neutrophils, lymphocytes and mononuclear cells (Figure 1B). The $12.5 \mathrm{mg} /$ $\mathrm{kg}$ magnesium isoglycyrrhizinate group (Figure 1C) underwent hepatocyte damage. However, compared with the model group, the injury and inflammatory cell infiltration were significantly alleviated. The changes were similar to those of the dexamethasone group (Figure 1D).

\section{Effects of magnesium isoglycyrrhizinate on MPO level in liver homogenate}

The MPO level in the liver homogenate of the model group was significantly higher than that of the normal control group, $(\mathrm{P}<0.01)$. The MPO levels in the liver homogenates of different magnesium isoglycyrrhizinate dose groups were significantly lower than that of model mice
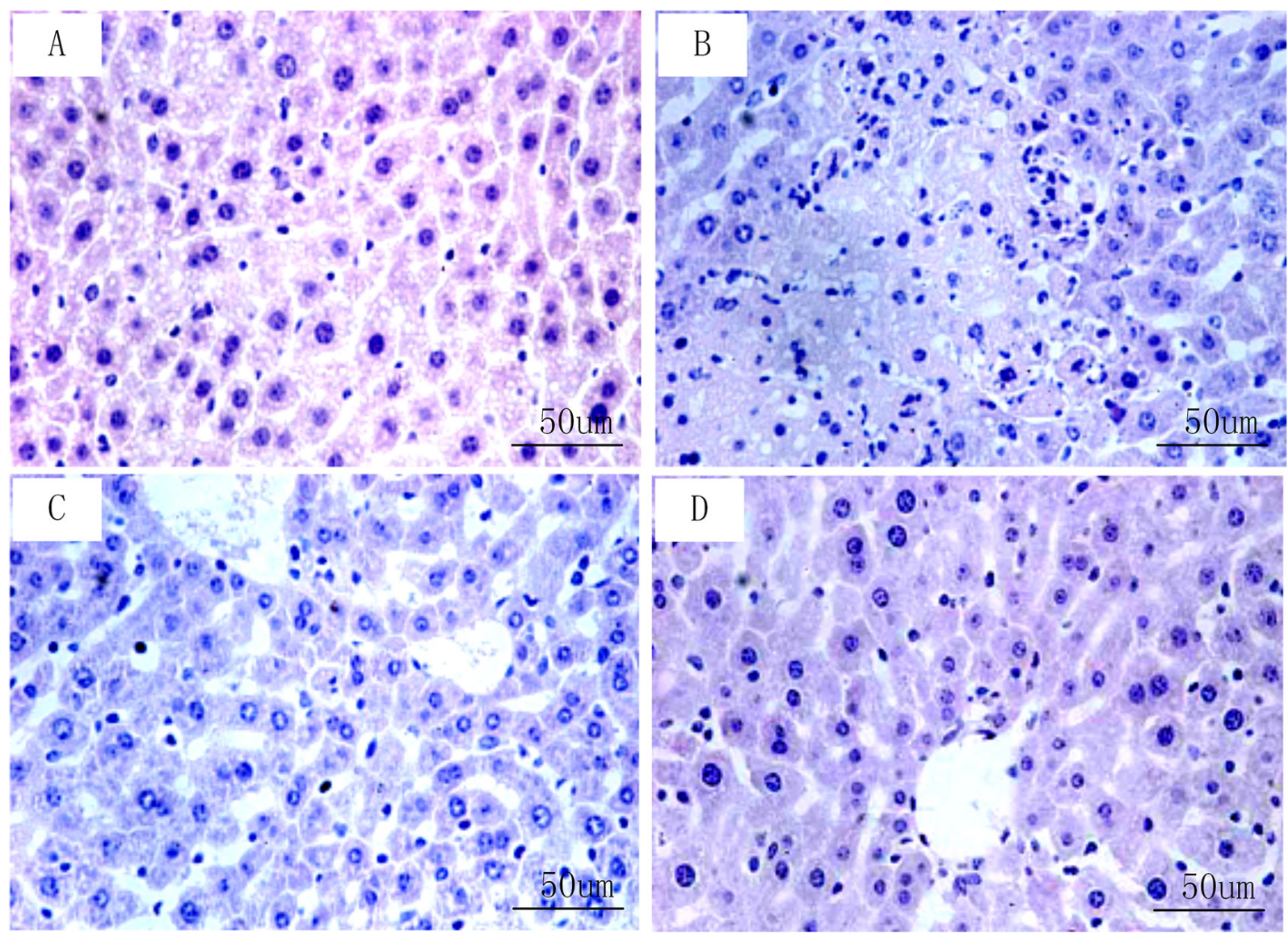

Fig. 1. Effects of magnesium isoglycyrrhizinate on liver pathology observed by HE staining. A: Normal control group in which hepatic lobules were intact and hepatocytes were arranged radially with the central vein as center; B: model group in which most hepatocytes in liver lobules were swollen, with obvious punctate and necrotic foci. A large number of neutrophils, lymphocytes and mononuclear cells infiltrated in the necrotic foci; $\mathrm{C}: \mathbf{1 2 . 5} \mathbf{~ m g} / \mathbf{k g}$ magnesium isoglycyrrhizinate group which underwent hepatocyte damage. However, compared with the model group, the injury and inflammatory cell infiltration were significantly alleviated. The changes were similar to those of the dexamethasone group; $D$ : dexamethasone group with a similar image to that of the normal control group. Magnification: '400. 
$(\mathrm{P}<0.05)$, similar to the level of the dexamethasone group (Figure 2), indicating that hepatic inflammation was significantly mitigated.

Effects of magnesium isoglycyrrhizinate on $M D A$ and SOD levels in liver homogenate MDA and SOD are crucial antioxidant enzymes. Compared with the normal control group, the MDA level in the liver homogenate of the model group was significantly higher $(\mathrm{P}<0.01)$, and the SOD level was significantly lower $(\mathrm{P}<0.01)$. Compared with the model group, the MDA levels in each dose group of magnesium isoglycyrrhizinate and the dexamethasone group were lower $(\mathrm{P}<0.01 ; \mathrm{P}<0.05)$, and the SOD levels were higher $(\mathrm{P}<0.01 ; \mathrm{P}<0.05)$ (Table 2$)$, suggesting that magnesium isoglycyrrhizinate exhibited similar antioxidative activity to that of dexamethasone.

\section{Effects of magnesium isoglycyrrhizinate on} plasma TNF- $\alpha$ and IFN- $\gamma$ levels

The levels of immunomodulatory cytokines TNF- $\alpha$ and IFN- $\gamma$ in the model group were significantly higher than those in the normal con-

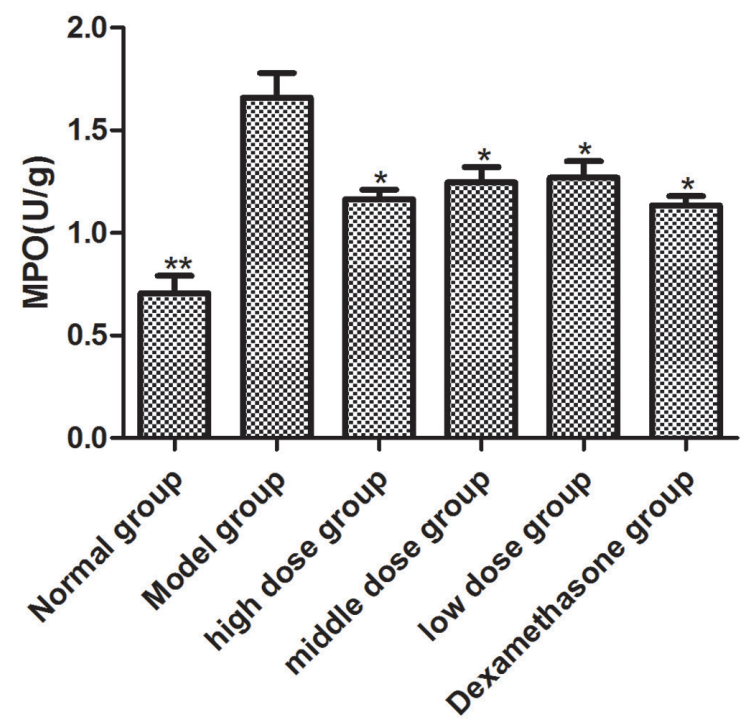

Fig. 2. Effects of magnesium isoglycyrrhizinate on MPO level of liver homogenate. Compared with model group, ${ }^{*} \mathbf{P}<0.05, * * \mathbf{P}<0.01$. trol group $(\mathrm{P}<0.01)$. The levels of TNF- $\alpha$ and IFN- $\gamma$ in different dose groups of magnesium isoglycyrrhizinate and the dexamethasone group were significantly lower than those of the model group $(\mathrm{P}<0.01 ; \mathrm{P}<0.05)$ (Table 3), suggesting that magnesium isoglycyrrhizinate had comparable immunomodulatory effects to those of dexamethasone.

\section{Effects of magnesium isoglycyrrhizinate on NP level in liver homogenate}

NP is a well-known marker of liver inflammation. The NP level of the model group significantly exceeded that of the normal group $(\mathrm{P}<0.01)$. Magnesium isoglycyrrhizinate at each dose and dexamethasone significantly reduced the NP level compared with that of the model group $(\mathrm{P}<0.01)$ (Figure 3), suggesting that they exerted similar anti-inflammatory effects.

\section{Discussion}

Compared with other toxin-induced liver injury models, the mouse model of ConA-induced

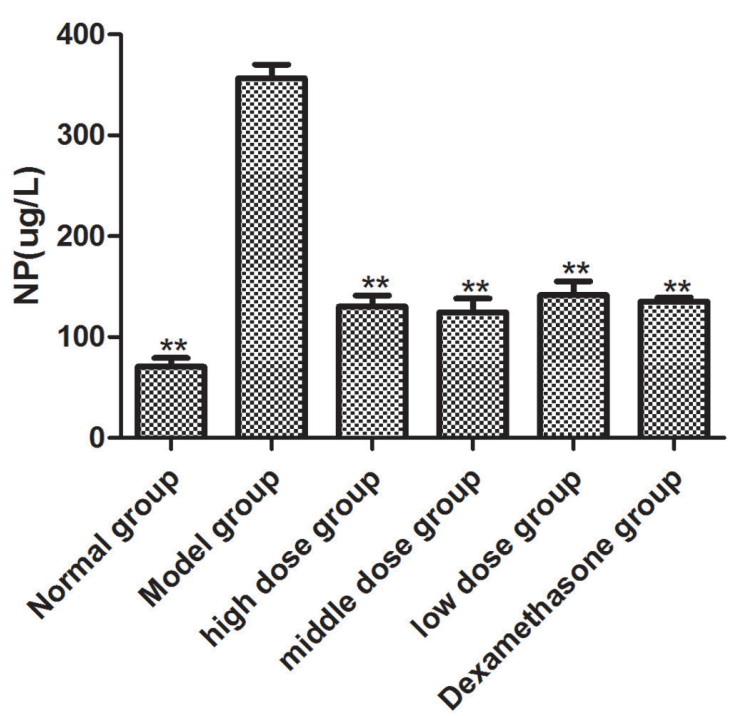

Fig. 3. Effects of magnesium isoglycyrrhizinate on NP level of liver homogenate. Compared with model group, $* * \mathbf{P}<0.01$. 
Table 2. Effects of magnesium isoglycyrrhizinate on MDA and SOD levels in liver homogenate

\begin{tabular}{lccc}
\hline Group & Dose $\mathbf{( m g / k g )}$ & MDA $(\mathbf{m m o l} / \mathbf{m g}$ Prot) & SOD (U/mg Prot) \\
\hline Normal control & & $29.82 \pm 1.62^{* *}$ & $344.70 \pm 13.00^{* *}$ \\
\hline Model & & $92.81 \pm 8.87$ & $168.87 \pm 11.11$ \\
\hline Magnesium isoglycyrrhizinate & 50 & $59.68 \pm 4.96^{* *}$ & $287.01 \pm 11.01^{*}$ \\
\hline & 25 & $57.48 \pm 4.66^{* *}$ & $295.97 \pm 11.05^{* *}$ \\
\hline Dexamethasone & 12.5 & $70.31 \pm 5.20^{* *}$ & $279.84 \pm 13.92^{*}$ \\
\hline
\end{tabular}

Compared with model group, ${ }^{*} \mathrm{P}<0.05, * * \mathrm{P}<0.01$.

immunological liver injury is more suitable for

tion, suggesting that prophylactic administration

Table 3. Effects of magnesium isoglycyrrhizinate on plasma TNF- $\alpha$ and IFN- $\gamma$ levels

\begin{tabular}{lccc}
\hline Group & Dose $(\mathrm{mg} / \mathrm{kg})$ & TNF- $\alpha(\mathrm{pg} / \mathrm{ml})$ & IFN- $\gamma(\mathrm{pg} / \mathrm{ml})$ \\
\hline Normal control & & $187.48 \pm 9.86^{* *}$ & $94.40 \pm 2.38^{* *}$ \\
\hline Model & & $498.65 \pm 17.27$ & $1786.73 \pm 24.30$ \\
\hline Magnesium isoglycyrrhizinate & 50 & $348.69 \pm 15.85^{*}$ & $577.70 \pm 17.38^{* *}$ \\
\hline & 25 & $301.77 \pm 12.49^{* *}$ & $466.53 \pm 15.68^{* *}$ \\
\hline Dexamethasone & 12.5 & $311.40 \pm 8.63^{* *}$ & $792.51 \pm 19.37^{* *}$ \\
\hline
\end{tabular}

Compared with model group, ${ }^{*} \mathrm{P}<0.05,{ }^{* *} \mathrm{P}<0.01$.

studying the pathological mechanism of human viral hepatitis and autoimmune liver disease as well as for screening applicable drugs [9], due to similar pathophysiological changes in the biochemical indices of liver function to those of human. Intravenous injection of ConA, a plant-derived lectin, may cause specific liver damage [10]. Injecting ConA into animals can elevate the levels of transaminases. Herein, serum ALT and AST activities increased $8 \mathrm{~h}$ after tail vein injection of ConA. Meanwhile, there were obvious necrotic foci where a large number of neutrophils, lymphocytes and mononuclear cells infiltrated. It has previously been proven that glycyrrhizin preparations were capable of combating viruses as well as reducing ALT and AST levels [11,12]. In this study, magnesium isoglycyrrhizinate significantly attenuated the serum ALT and AST activities of mice with liver injury, and relieved liver pathological damages such as necrosis and inflammatory cell infiltra- of magnesium isoglycyrrhizinate alleviated ConA-induced liver injury.

ConA is a mitogen that can activate $\mathrm{T}$ lymphocytes and stimulate $\mathrm{T}$ helper cells and macrophages to produce cytokines such as TNF- $\alpha$ and IFN- $\gamma$. TNF- $\alpha$ leads to hepatocyte apoptosis, possibly by binding its receptor to directly initiate the apoptotic signaling pathway, or by facilitating the accumulation of neutrophils in the liver to release protease or oxygen free radicals. Thus, TNF- $\alpha$ plays a key role in models of ConA-induced liver injury $[13,14]$. After antibody against TNF- $\alpha$ is administered, the apoptosis of liver tissue in animals can be almost completely restored and the degree of injury can be significantly alleviated $[15,16]$. Besides, IFN- $\gamma$ can promote Kupffer cells in the liver to participate in inflammatory reaction, and pre-administration of antibody against IFN- $\gamma$ can protect against ConA-induced immunological liver injury [17]. Consistently, this study showed that 
plasma TNF- $\alpha$ and IFN- $\gamma$ levels increased $8 \mathrm{~h}$ after tail vein injection of ConA. Magnesium isoglycyrrhizinate significantly reduced the levels of TNF- $\alpha$ and IFN- $\gamma$, indicating that it played a protective role by modulating immunity and down-regulating cytokine levels.

As one of the sensitive indices for evaluating the activation degree of immune system in vivo [18], $\mathrm{NP}$ is often used to measure inflammatory activity. In the inflammatory state, a variety of inflammatory cells release a large amount of oxygen free radicals, attack polyunsaturated fatty acids on the cell membrane, and trigger lipid peroxidation, causing damage to cells and aggravating liver damage. MDA is the final product of lipid peroxidation, resulting in damage to the cell membrane and loss of function. Therefore, measuring the MDA level can indirectly reflect the extent of cell damage. SOD, a scavenger of superoxide anion free radicals, can inhibit free radical-initiated lipid peroxidation. It plays a vital role in the oxidant-antioxidant balance, and its activity indirectly reflects the capacity of eliminating oxygen free radicals. Herein, the levels of NP and MDA in the model group were significantly higher than those of the normal control group, and the activity of SOD was lower. However, magnesium isoglycyrrhizinate reduced the NP and MDA levels and increased the SOD activity, suggesting that it exerted hepatoprotective effects through an antioxidant mechanism.

Activated neutrophils are known to cause tissue damage by releasing reactive oxygen species $\left(\mathrm{O}^{2-}\right)$ and other toxic substances. As an important peroxidase released by neutrophil azurophilic granules, MPO can mediate inflammatory reactions and immune responses, thus being a key marker of neutrophil infiltration [19]. In this study, tail vein injection of ConA significantly raised the liver MPO level, suggesting evident inflammatory cell infiltration in liver tissues. In contrast, magnesium isoglycyrrhizinate reduced the liver MPO level and the local infiltration of inflammatory cells, and alleviated the resulting tissue damage.

In summary, magnesium isoglycyrrhizinate down-regulated the levels of pro-inflammatory cytokines such as TNF- $\alpha$ and IFN- $\gamma$, resisted oxidation and exerted hepatoprotective effects by modulating immunity. The findings provide valuable experimental basis for treating liver diseases such as immunological liver injury by using this drug. Nevertheless, this study has limitations. Only an animal model was tested, so further clinical studies using large-size samples are in need before the clinical use of magnesium isoglycyrrhizinate.

\section{Authors' contribution}

Zhengyan Jiang (Investigation; Methodology; Writing - original draft)

Liang Zheng (Conceptualization; Supervision; Writing - review \& editing)

\section{Conflict of interest}

There was no conflict in this work.

\section{Acknowledgement}

This study was not financially supported.

\section{Abbreviations}

$\mathrm{ALT}=$ alanine transaminase

$\mathrm{AST}=$ aspartate transaminase

Con $\mathrm{A}=$ concanavalin $\mathrm{A}$

IFN- $\gamma=$ interferon $-\gamma$

$\mathrm{MDA}=$ malondialdehyde

$\mathrm{MPO}=$ myeloperoxidase

$\mathrm{NP}=$ neopterin

$\mathrm{OD}=$ optical density

$\mathrm{PBS}=$ phosphate buffered saline

$\mathrm{SOD}=$ superoxide dismutase

TNF- $\alpha=$ tumor necrosis factor- $\alpha$ 


\section{References}

1. Zhou YX, Mo SL, Wang RH, Liu WF, Liu JC, Wang JQ, et al. Corn baked by burning coal triggered overexpression of osteopontin in hepatocytes of rats following fluorosis. Toxicol Ind Health. 2012;28(3):195-202. DOI: $10.1177 / 0748233711410905$

2. Wang J, Zhao P, Guo H, Sun X, Jiang Z, Xu L, et al. Serum IL-33 levels are associated with liver damage in patients with chronic hepatitis C. Mediators Inflamm. 2012;2012:819636. DOI: 10.1155/2012/819636

3. Ingawale DK, Mandlik SK, Naik SR. Models of hepatotoxicity and the underlying cellular, biochemical and immunological mechanism(s): a critical discussion. Environ Toxicol Pharmacol. 2014;37(1):118-33. DOI: 10.1016/j.etap.2013.08.015

4. Rijckborst V, Sonneveld MJ, Janssen HL. Review article: chronic hepatitis B - anti-viral or immunomodulatory therapy? Aliment Pharmacol Ther. 2011;33(5):50113. DOI: 10.1111/j.1365-2036.2010.04555.x

5. de Groen RA, Boltjes A, Hou J, Liu BS, McPhee F, Friborg $\mathrm{J}$, et al. IFN- $\lambda$-mediated IL-12 production in macrophages induces IFN- $\gamma$ production in human NK cells. Eur J Immunol. 2015;45(1):250-9. DOI: 10.1002/ eji.201444903

6. Yang Q, Zhao X, Zang L, Fang X, Zhao J, Yang X, et al. Anti-hepatitis B virus activities of $\alpha$-DDB-FNC, a novel nucleoside-biphenyldicarboxylate compound in cells and ducks, and its anti-immunological liver injury effect in mice. Antiviral Res. 2012;96(3):333-9. DOI: 10.1016/j.antiviral.2012.10.003

7. Erhardt A, Biburger M, Papadopoulos T, Tiegs G. IL-10, regulatory $\mathrm{T}$ cells, and Kupffer cells mediate tolerance in concanavalin A-induced liver injury in mice. Hepatology. 2007;45(2):475-85. DOI: 10.1002/ hep. 21498

8. Xie C, Li X, Wu J, Liang Z, Deng F, Xie W, et al. Anti-inflammatory Activity of Magnesium Isoglycyrrhizinate Through Inhibition of Phospholipase A2/Arachidonic Acid Pathway. Inflammation. 2015;38(4):1639-48. DOI: 10.1007/s10753-015-0140-2

9. Tiegs G, Hentschel J, Wendel A. AT cell-dependent experimental liver injury in mice inducible by concanavalin A. J Clin Invest. 1992;90(1):196-203. DOI: 10.1172/JCI115836

10. Gantner F, Leist M, Jilg S, Germann PG, Freudenberg MA, Tiegs G. Tumor necrosis factor-induced hepatic DNA fragmentation as an early marker of $\mathrm{T}$ cell-dependent liver injury in mice. Gastroenterology. 1995;109(1):166-76. DOI: 10.1016/00165085(95)90282-1

11. Yu JJ, Zhang CS, Coyle ME, Du Y, Zhang AL, Guo $\mathrm{X}$, et al. Compound glycyrrhizin plus conventional therapy for psoriasis vulgaris: a systematic review and meta-analysis of randomized controlled trials. Curr Med Res Opin. 2017;33(2):279-87. DOI: 10.1080/03007995.2016.1254605

12. van Rossum TGJ. In: Glycyrrhizin treatment for Chronic Hepatitis C. Erasmus University Rotterdam. 2000; retrieved from http://hdl.handle.net/1765/20441.

13. Wang HX, Liu M, Weng SY, Li JJ, Xie C, He HL, et al. Immune mechanisms of Concanavalin A model of autoimmune hepatitis. World J Gastroenterol. 2012;18(2):119-25. DOI: 10.3748/wjg.v18.i2.119

14. Li WW, Yu JY, Xu HL, Bao JK. Concanavalin A: a potential anti-neoplastic agent targeting apoptosis, autophagy and anti-angiogenesis for cancer therapeutics. Biochem Biophys Res Commun. 2011;414(2):282-6. DOI: $10.1016 /$ j.bbrc.2011.09.072

15. Jakubowski A, Sternak M, Jablonski K, Ciszek-Lenda M, Marcinkiewicz J, Chlopicki S. 1-Methylnicotinamide protects against liver injury induced by concanavalin A via a prostacyclin-dependent mechanism: A possible involvement of IL-4 and TNF- $\alpha$. Int Immunopharmacol. 2016;31:98-104. DOI: 10.1016/j.intimp.2015.11.032

16. Li J, Gong YM, Wu J, Wu WJ, Cai W. Anti-tumor necrosis factor- $\alpha$ monoclonal antibody alleviates parenteral nutrition-associated liver disease in mice. JPEN J Parenter Enteral Nutr. 2012;36(2):219-25. DOI: 10.1177/0148607111424412

17. Nicoletti F, Zaccone P, Xiang M, Magro G, Di Mauro M, Di Marco R, et al. Essential pathogenetic role for interferon (IFN-)gamma in concanavalin A-induced $\mathrm{T}$ cell-dependent hepatitis: exacerbation by exogenous IFN-gamma and prevention by IFN-gamma receptor-immunoglobulin fusion protein. Cytokine. 2000;12(4):315-23. DOI: 10.1006/cyto.1999.0561

18. Capuron L, Geisler S, Kurz K, Leblhuber F, Sperner-Unterweger B, Fuchs D. Activated immune system and inflammation in healthy ageing: relevance for tryptophan and neopterin metabolism. Curr Pharm Des. 2014;20(38):6048-57. DOI: 10.2174/13816128206661 40317110217 
19. Ceccarelli G, Brenchley JM, Cavallari EN, Scheri GC, Fratino M, Pinacchio C, et al. Impact of High-Dose Multi-Strain Probiotic Supplementation on Neurocog- nitive Performance and Central Nervous System Immune Activation of HIV-1 Infected Individuals. Nutrients. 2017;9(11):E1269. DOI: 10.3390/nu9111269 